\title{
Non-Oral Drug Delivery in Parkinson's Disease: Current Applications and Future
}

\author{
(D) Meliha GÜNEŞ, (D) Sinem Yaprak KARAVANA* \\ Ege University, Faculty of Pharmacy, Department of Pharmaceutical Technology, İzmir, Türkiye
}

\begin{abstract}
Parkinson's disease (PD) is a type of movement disorder that affects the ability to perform daily activities. It is considered that 1 million people in the U.S. and more than 10 million people worldwide live with PD. It is a chronic and progressive disease, so symptoms worsen over the time. Patients experience motor symptoms such as tremors, stiffness and slow motion, and non-motor symptoms such as sleep problems, constipation, anxiety, depression and fatigue. Dopaminergic drugs are critical for treating motor symptoms in PD. Levodopa (L-DOPA) is the "gold standard" medication for the control of motor symptoms. Because of the progression of the disease, the effectiveness of oral L-DOPA decreases over time and motor fluctuations such as "delayed ON", "no ON" and unpredictable "ON-OFF" periods appear. These motor fluctuations affect the quality of life of the patient at a high rate and the patient has problems in fulfilling his daily morning routines. Gastrointestinal (Gl) problems, as the common non-motor symptom, are the most important cause of motor fluctuations that occur because of inadequate oral treatment with the progression of PD. When oral treatments are not sufficient, non-oral treatments that are not affected by Gl problems are required. In this review, the treatment strategies, developed and approved non-oral drug delivery systems in the early and advanced stages of PD are emphasized.
\end{abstract}

Keywords: Parkinson's disease, oral and non-oral treatment, motor and non-motor fluctuations

\section{INTRODUCTION}

Parkinson's disease (PD) is the most common neurodegenerative movement disorder that can affect the ability to perform daily activities. 'It is considered that 1 million people in the U.S. and more than 10 million people worldwide have PD. PD is usually diagnosed in people over the age of 55. Although it is rare, it can also be seen in the young population between the ages of 21-45. The disease is called late-onset, when diagnosed in older people, and young-onset, when diagnosed in the young population. ${ }^{2}$

PD is a chronic and progressive disease. Motor and nonmotor symptoms are seen in these patients. However, it is characterized by motor symptoms associated with movement. These symptoms are rhythmic shaking tremors, stiffness or rigidity of the muscles and slowness of the movement (bradykinesia). Movements are controlled by neurons in the brain and messages are transmitted to each other and to the rest of the body by chemicals called neurotransmitters. Dopamine, a neurotransmitter that control movement, is produced in substantia nigra area of the brain. In PD, 70-80\% of dopamine-producing cells disrupt by stages and are lost, which called neurodegeneration. The damage to neurons causes low levels of dopamine in the part of the brain that controls balance and movement. When neurons do not pass on brain messages properly, their movements have not been controlled smoothly and the motor symptoms of PD appear. In addition to motor symptoms, non-motor symptoms related to PD can occur in patients. Non-motor symptoms are sleep problems, constipation, depression, anxiety, and fatigue. For many of these non-motor symptoms, definitive clinicopathological correlations are still not fully understood. ${ }^{3}$ Dopaminergic drugs are critical for treating motor symptoms in PD. Levodopa (L-DOPA) is known as the "gold standard" for the control of motor symptoms in PD. Because of the progression of PD, effectiveness of oral L-DOPA decreases over time. ${ }^{4}$ It has been reported that in 5-10 years, patients treated L-DOPA will develop motor fluctuations and dyskinesias in $70-80 \%{ }^{5}$ The fluctuations in motor functions 
are due to $\mathrm{ON}$ responses (good antiparkinsonian effect) and OFF responses (the symptoms are not efficiently controlled) seen just before the next dose of L-DOPA. In the ON period, patients can fully move and function independently, and the patient is unable to function such as move, talk, smile as easily during the OFF period. These motor fluctuations can occur diversely. These are foreseeable end-of-dose "wearing OFF" phenomena, peripheral problems such as "delayed ON" or "no ON", and unpredictable "ON-OFF" periods. The delayed effect of oral medications causes an early morning OFF period. ${ }^{6,7}$ This condition affects quality of life of the patients at a high rate and the patient has problems in fulfilling his daily morning routines. The results of an international multicenter study of EUROPAR, a partner of the European Parkinson's Disease Association, show that the incidence of OFF period is $60 \%$ even in patients undergoing optimized PD therapy. ${ }^{8} \mathrm{~L}-\mathrm{DOPA}$ dose is usually increased to manage these problems. However, increasing L-DOPA dose can cause involuntary movements or painful dyskinesia. Gastrointestinal (Gl) problems, as a common non-motor symptom, are the most important cause of motor fluctuations that occur because of inadequate oral treatment with the progression of PD. Dysphagia, gastric dysfunction, colonic dysmotility, small-intestine motility, and delayed gastric emptying (GE) can be considered Gl problems. When oral therapies are not insufficient, alternative drug delivery systems that are not affected by Gl problems are necessary, which are known as non-oral treatments. Guidelines published in 2017 at the National Institute for Health and Care Excellence mention that non-oral treatments will be safe, important and effective for PD treatment.9.10 In this review, importance of non-oral therapy in PD treatment is emphasized. It also includes available non-oral drug delivery systems and current studies of non-oral formulations.

\section{METHODS}

We used the websites of the American Parkinson Disease Association and the European Parkinson Disease Association for this review. Additionally, references for this review have been identified through PubMed, ScienceDirect and Google Academic using the terms "Parkinson's disease", "Parkinson's disease treatment strategy" and "Non-oral treatment of Parkinson's disease". We primarily selected articles published between 2000 and 2020. Only publications in English were evaluated. We evaluated more than 200 citations, of which 81 are included in this review.

\section{Current oral treatment options for Parkinson's disease}

There is no definite cure for PD, but the medicines used in treatments can provide important symptomatic control of the motor symptoms. Current pharmaceutical strategies for the control of symptoms are L-DOPA, catechol-O-methyl transferase (COMT) inhibitors, dopamine agonists, monoamine oxidase B (MAO-B) inhibitors, anticholinergic, and amantadine medications. ${ }^{10}$

L-DOPA is a medicine used since 1970 to treat PD and is still most effective for symptomatic treatment. It is effective in the early stages of PD but remains effective as PD progresses without intolerance developing over time. L-DOPA is routinely used along with a DOPA-decarboxylase inhibitor to reduce some treatment complications, prolong half-life, and increase L-DOPA availability to the brain.11 However, after long-term use of L-DOPA oral formulations, problems such as motor and non-motor fluctuations and L-DOPA-induced dyskinesia can be observed because of pharmacokinetic properties of L-DOPA. Patients do not experience any fluctuations in motor or non-motor symptoms during the first years of L-DOPA use. Patients begin to aware of these fluctuations after 2-5 years of L-DOPA use. In this way, as the disease progresses, patients must make frequent adjustments to the dosage regimen and they should use L-DOPA more frequently due to the shortened effect time and reduced effect. ${ }^{12}$ Increasing the dose and frequency of L-DOPA to control of motor symptoms may provide some improvement, but involuntary movements and painful dyskinesia may occur due to the high plasma concentration of L-DOPA. Dyskinesia can cause the problems in walking and balance; therefore, patients may have difficulties in social life. Additionally, in the later stages of PD, patients become completely dependent on care, and those caring for their care has a heavy social responsibility, both socially and economically. 13,14

Since motor fluctuations greatly affect the course of the disease, clinicians occasionally have difficulty managing the disease. After 5 years of L-DOPA treatment, approximately $50 \%$ of patients experience wearing off, and this rate rises to about $80 \%$ after 10 years. ${ }^{15}$ Clinicians should choose the appropriate PD medicines to manage symptoms effectively and improve the patient's quality of life. The most important reason for fluctuations in the use of oral PD medications are Gl dysfunctions such as slow GE, irregular jejunal absorption, and competition with dietary amino acids in the areas of absorption. ${ }^{16,17}$

COMT inhibitors are drugs that inhibit the enzyme COMT that acts on dopamine breakdown and extend the duration of L-DOPA activity. Doctors use them along with L-DOPA to treat the motor symptoms of PD. ${ }^{18}$ Because they prolong L-DOPA duration of action by increasing half-life and delivery to the brain. In some patients, COMT inhibitors provide control of motor symptoms by reducing off-time compared with standard L-DOPA/DOPA decarboxylase inhibitor combinations. ${ }^{19}$ Tablet formulations of COMT inhibitors are available on the market. Although they are able to improve motor function in some patients, they are not prescribed alone because they offer a limited effect on PD symptoms. Entacapone and tolcapone, approved COMT inhibitors, are reversible COMT inhibitors approved for treating $P D$. A third COMT inhibitor, opicapone, is available in Europe but has not yet been approved by the Food and Drug Administration (FDA). Each of these COMT inhibitors has problems in terms of pharmacokinetics, pharmacodynamics, clinical efficacy or safety. Additionally, their elimination half-lifes are approximately 2-3 hours. ${ }^{20}$ 
The most common adverse effects associated with the addition of COMT inhibitors to carbidopa/L-DOPA treatment are strengthening the dopaminergic effects of drugs, such as nausea, dyskinesia, orthostatic hypotension, sleep disorders, hallucinations, and vomiting. L-DOPA dose adjustment must avoid these events. Dark yellow or orange urine discoloration is related to the colour of the COMT inhibitors and their metabolites. Entacapone from COMT inhibitors is preferred as the first-line treatment in patients with PD. Because tolcapone causes hepatotoxicity. The descriptions of acute, fatal fulminant hepatitis and potentially fatal neurological reactions in association with tolcapone led to the suspension of its marketing authorization in Europe and Canada. In many other countries, use of the drug is restricted to patients, who are not responding to other therapies. If tolcapone is used in PD treatment, proper monitoring of liver function, and liver enzymes is required during the first six to eight months of the treatment. ${ }^{21,22}$

MAO-B inhibitors have been used for treating PD as both early monotherapy and combined therapy in patients with the more advanced disease. ${ }^{23}$ Selegiline and rasagiline are selective MAO-B inhibitors approved for PD treatment. ${ }^{24}$ Both selegiline and rasagiline were originally developed as antidepressants. However, low and medium doses of selegiline required to provide an irreversible MAO-B inhibition have not had antidepressant activity. The most important differences between these two active substances are their metabolism, their interaction with cytochrome (CYP) P450 enzymes and their molecular biological/genetic level properties. ${ }^{25}$ Amphetamine metabolites occur because of the metabolism of selegiline with CYP enzymes. These metabolites can occur after oral use and can cause sleep problems in patients. ${ }^{24}$

The oral bioavailability of selegiline is about $10 \%$. This low bioavailability has led to the development of different nonoral drug delivery systems such as transdermal, buccal, and nasal. ${ }^{26,27}$ Another MAO-B inhibitor is rasagiline and because of the metabolism of rasagiline, unwanted metabolites such as amphetamine-like metabolites do not form. ${ }^{18}$ Studies have shown that amphetamine-like metabolites occur only in the plasma of patients with PD during the use of selegiline, and not during chronic rasagiline therapy. ${ }^{28,29}$ Additionally, rasagiline administered orally is rapidly absorbed from Gl tract and reaches the highest plasma concentrations within an hour. Rasagiline's oral bioavailability is about $36 \%$ due to its high hepatic first-pass metabolism. ${ }^{30}$

"Cheese reaction", which is a serious side effect, occurs especially when non-selective MAO inhibitors are administered with certain foods such as cheese and drugs such as decongestants. Because of this reaction, hypertensive crisis, palpitations, tachycardia, blurred vision, arrhythmias, and other sympathomimetic problems can be seen. The "cheese reaction" occurs particularly, when older MAO inhibitors are administered with biogenic amine-like substances such as decongestants or high dietary tyramine (more than $500 \mathrm{mg}$ per day). Although there are clinical pharmacology and safety data showing that rasagiline and selegiline are selective $\mathrm{MAO}-\mathrm{B}$ inhibitors, concerns remain regarding interactions with tyramine and the potential for hypertensive crisis. Despite being rare, cases of the "cheese reaction" have been informed during treatment with selegiline. It has been stated that normal dietary tyramine for both selegiline and rasagiline does not cause clinically meaningful interactions, but taking more than $150 \mathrm{mg}$ tyramine per day may increase the risk. ${ }^{25}$ In the study of Goren et al. ${ }^{29}$, rasagiline at the recommended therapeutic dose of $1 \mathrm{mg} /$ day provides a selective MAO-B inhibition. Simultaneously, it has also been noted that, when rasagiline is used at doses $>2 \mathrm{mg} /$ day, its selectivity for MAO-B decreases and tyramine sensitivity increases. ${ }^{29}$

Dopamine agonists demonstrate antiparkinsonian effects by directly acting on dopamine receptors and mimicking the endogenous neurotransmitter. Oral L-DOPA/DOPA decarboxylase inhibitor application is inevitably necessary with the advance of PD. In the long term, chronic administration of oral L-DOPA formulations in a fixed combination with inhibitors of the main metabolizing L-DOPA enzymes results in the onset of so-called motor complications. When the disease progresses, the duration of L-DOPA response shortens in addition to the short plasma L-DOPA half-life. ${ }^{31} \mathrm{~L}$-DOPA converts to dopamine in both the center nervous system and the periphery. To increase the bioavailability of L-DOPA and decrease its side effects, it is often administered along with peripheral decarboxylase inhibitors (such as carbidopa and benserazide). Dopamine decarboxylase inhibitors prevent the conversion of L-DOPA to dopamine in the periphery, allowing for more L-DOPA to cross the brain-blood barrier. ${ }^{32}$

Compared to L-DOPA, dopamine receptor agonists do not require the enzymatic conversion to an active metabolite, and do not have potentially toxic metabolites. However, they do not compete with other substances for their active transport across the blood and blood-brain barrier, and are not depended on the functional capacity of nigrostriatal neurons. ${ }^{18}$ Dopamine agonists are classified as ergot or non-ergot types, and these active agents have essential differences associated with receptor affinities. Bromocriptine and cabergoline, as ergot derivatives, are dopamine agonists and they are not commonly used for treating PD. Ropinirole and pramipexole rotigotine are non-ergot-derived dopamine agonists, which are approved for PD therapy. ${ }^{33}$ Apomorphine is the most potent dopamine agonist, but it effectively stimulates both D1 and D2 receptors like dopamine. However, due to some limitations, apomorphine cannot be used as an oral drug. ${ }^{34}$

Anticholinergic agents recently used to treat PD are benztropine and trihexyphenidyl. Since these drugs non-selectively block cholinergic receptors in the body, some side effects are seen. There are some hesitations about the use of these drugs for this reason. When selective cholinergic receptor antagonists were tried, significant benefits could not be obtained in PD treatment. Anticholinergics can alleviate dystonia and tremors caused by wearing off. However, it has no significant effect on other PD symptoms. ${ }^{35}$ 


\section{Dysfunctions of gastrointestinal system in patients with $P D$}

\section{Dysphagia}

Chewing and swallowing functions require regularly contracting and relaxing of many muscles. Therefore, it is inevitable that dysphagia is common in patients with PD. Dyspgahia is a problem that reduces quality of life and obstructs intake of the medication and increases the risk of aspiration, which is the cause of death of most patients in PD. PD-related dysphagia is not fully understood. Nevertheless, dopaminergic and nondopaminergic mechanisms are effective in the development of dysphagia in PD. ${ }^{36}$

Lately, results of the studies disclosed that the dysphagia prevalence based on subjective conclusions, in patients with $P D$ is $35 \%$ and rises to $82 \%$ by taking objective measures of swallowing dysfunction into account. ${ }^{37}$ Aydogdu et al. ${ }^{35}$ evaluated the dysphagia prevalence with the Videofluoroscopic Swallowing Study (VFSS) using the guidelines of the United Kingdom Parkinson's Disease Brain Bank. In this study, VFSS evaluation was performed on 23 patients with PD and 16 of the total sample were diagnosed with dysphagia. ${ }^{35}$ Some clinical predictors should be considered when evaluating a patient with PD for the presence of dysphagia. For example; in patients with $\mathrm{PD}$, weight loss without any reason or a body mass index below 20 is highly indicative of dysphagia. It is stated that $20 \%$ of patients develop malnutrition during PD. Another predictor of dysphagia and aspiration pneumonia is sialorrhea or drooling. ${ }^{37,38}$

\section{Drooling}

Drooling has many negative effects on quality of life, such as social embarrassment, decreased oral hygiene, bad breath, increased oral bacteria, difficulty speaking and eating, and increased risk of aspiration pneumonia. ${ }^{39}$ There are no standard description and criterion for the diagnosis of drooling in patients with PD. For this reason, the prevalence forecast varies. Leibner et al. ${ }^{37}$ conducted a questionnaire study on the drooling problem with 58 patients with PD and 51 healthy volunteers. In the end of the study, when patients with PD and control groups were compared, the rate of drooling was $59 \%$ and $14 \%$, respectively. ${ }^{37}$ Müller et al. ${ }^{38}$ managed a study to examine the emergence and severity of autonomic and sensory symptoms in 207 newly diagnosed, untreated patients with PD and 175 healthy volunteers. The most obvious difference was observed in drooling, which was present in $42 \%$ of patients with PD but just $6 \%$ of the control group.

\section{Gastric emptying}

Disrupted GE (gastroparesis) is a common problem in patients with PD. In gastroparesis, patients experience symptoms such as abdominal discomfort or postprandial bloating, nausea, early satiety and weight loss. ${ }^{9}$ It is thought that the cause of delayed and motor fluctuations in PD is delayed GE. ${ }^{39}$

Tanaka et al. ${ }^{40}$ conducted a study with three groups. These groups were 20 patients with PD with newly diagnosed, untreated; treated with L-DOPA for a long time, advanced-stage 40 patients with PD; 20 healthy volunteers. The half-emptyting time $\left(T_{1 / 2}\right)$ of healthy volunteers, newly diagnosed untreated and long-treated patients were found to be $86 \mathrm{~min}, 122 \mathrm{~min}$, and $125 \mathrm{~min}$, respectively. Goetze et al. ${ }^{41}$ conducted a study with 36 patients with PD (divided into two as mild and advance) and 22 healthy volunteers. Because of this study, $97 \%$ of patients with PD had delayed GE. $T_{1 / 2}$ was found to be significantly longer in patients with PD compared in the control group. (169 vs. 107 $\mathrm{min})$. Delayed GE was associated with degree of the disease. GE was found 149 and 196 min for patients with mild and advanced $\mathrm{PD}$, respectively..$^{41}$ Unger et al..$^{42}$ subjected 20 healthy volunteers, 21 drug-naive and early-stage patients with PD and 18 patients with PD treated with dopaminergic medicines to $13 \mathrm{C}$ octanoate breath test to determine the duration of GE. Because of the study, it was observed that GE test (1/2) differs significantly between the groups. GE test (1/2) was found in control, drug-naive, early-stage patients with $P D$ and treated patients with PD, $123.3 \mathrm{~min} \pm 16.6,166.6 \mathrm{~min} \pm 32.4$, and 203.6 $\min \pm 46.8$, respectively. ${ }^{42}$ Most of these studies reported significantly increased the GE test in the PD group compared with the controls.

\section{Small intestinal bacterial overgrowth (SIBO)}

SIBO and changing gut microbiota raise doubts about the effectiveness of oral drug therapy in PD. ${ }^{41}$ Recent studies showed that incidence of SIBO is high in PD. Additionally, GI symptoms and worsening of motor functions in $\mathrm{PD}$ have been reported to be related to SIBO.$^{43}$ Fasano et al. ${ }^{44}$ showed that patients with PD and SIBO have more serious motor fluctuations (off time, delayed on-time, and non-on-time) than those without SIBO.

\section{Colonic dysmotility}

One of the most important Gl problems observed in PD is decreased bowel movement. However, many PD drugs, such as anticholinergics and dopamine agonists, have been shown to cause constipation. ${ }^{39}$

In the study by Cheon et al. ${ }^{45}$, the rate of constipation in patients with PD is $65.8 \%$. In a survey study, the rates of difficulty in defecation in patients with PD and control group were reported as $59 \%$ and $20.9 \%$, respectively. In the same study, the rate of laxative prescribing was reported as 29.9\% and 9.5\%, respectively.

\section{Non-oral treatment necessity in $P D$ treatment}

Although orally administered L-DOPA is considered as "gold standard" drug for the control of motor symptoms in PD, the duration of benefit is seen to decreases in use long-term at an oral dose of L-DOPA. ${ }^{4}$ Patients begin experiencing fluctuations in motor function in the later stages of PD. Due to the late effects of oral medications, the early morning OFF-periods are the most challenging situation in PD. This problem can complicate the patient's daily morning routines and seriously affect their quality of life. ${ }^{7}$ As the disease progresses, the most important reason for oral treatment failure and motor fluctuations are the above-mentioned Gl problems. Dysfunctions in the Gl system occur at all levels of PD and this cause motor fluctuations in the advanced stages of PD, which make management of the disease difficult. ${ }^{46}$ Especially, dysphagia may induce silent 
aspiration and delayed GE. Problems such as delayed "on" and non "on" responses may arise due to gastroparesis in PD's oral dopaminergic treatment. ${ }^{7.16} \mathrm{Gl}$ problems, such as gastroparesis, which occur in $70-100 \%$ of patients, can decrease the effectiveness of oral medications by delaying their absorption and delivery into the bloodstream. ${ }^{16}$ Delayed $\mathrm{ON}$ and even dose failure, which causes motor fluctuations, may occur because of inadequate levels of medication plasma levels. ${ }^{47,48}$ Besides, it has been stated in recent studies that the pathological process of PD can be managed and even started by the intestinal microbiota through the intestinal-brain axis. ${ }^{49,50}$ Additionally, studies have shown that bacterial metabolites that may affect the enteric nervous system differ between patients with PD and healthy control groups. ${ }^{51}$ At the same time, it has been indicated that previous studies have that some PD drugs may change the microbiota content. The increasing recognition of multilevel $\mathrm{Gl}$ dysfunction in patients with $\mathrm{PD}$ has contributed to the development of non-oral methods for treating PD's motor and non-motor symptoms. ${ }^{46}$

\section{Current studies on non-oral formulations}

The liquid intranasal rotigotine is formulated of a pharmaceutically satisfactory acid addition salt of rotigotine and $\alpha$-cyclodextrin. $\alpha$-Cyclodextrin is used to predominantly stabilize rotigotine hydrochloride used. A formulation for intranasal use of rotigotine has been developed for therapy in PD and restless leg syndrome. The formulation underwent two phase 2 studies to assess efficacy, safety, and tolerability in a randomized, double-blind, placebo-controlled, and proofof-concept manner. However, the results of these studies did not show improvement in secondary outcome measures such as a change in Unified Parkinson's Disease Rating Scale III (UPDRS III) administration administration and "OFF" reversals. Development of the drug was discontinued. ${ }^{52}$

Priano et al. ${ }^{53}$ completed a pilot study on a new preparation of apomorphine, which was included in microemulsion and administration via the transdermal route (APO-MTD). Twentyone patients were treated and the results obtained showed that APO-MTD delivered an average of $5.1 \mathrm{~h}$ of therapeutic plasma levels, improved the UPDRS III scores, and reduced the overall length of "OFF" periods. However, as promising as this treatment may seem, because of the time taken of $1 \mathrm{~h}$ to reach therapeutic concentrations, APO-MTD may not be the "ideal" treatment for the rapid relief of the "OFF" periods suffered by patients with PD.

The sublingual formulation of the D2-D3 agonist piribedil, S90049, was designed to abort "OFF" episodes in PD. A phase 2, double-blind, randomized, and placebo-controlled study showed superiority of S90049 in UPDRS III post-application in advanced-stage patients with PD. Additionally, the switch from "OFF" to "ON" was significantly greater in patients using S90049 inhalation than in placebo. Despite these results, no further activity has been reported since $2010 .{ }^{54}$

Sintov et al. ${ }^{55}$ have suggested that transdermal L-DOPA administration can be effective to provide continuous dopaminergic stimulation. Considering that L-DOPA is insoluble in most solvents and has limited permeability through the skin, a modern self-assembling nanomicellar system with 2\% L-DOPA and $1 \%$ carbidopa has been developed. Because of in vitro tests and in vivo studies in rabbits, it has been observed that transdermal permeability and systemic absorption of L-DOPA from the skin increased significantly through this formulation developed.

Non-oral formulations are required because of high liver metabolism and poor oral bioavailability of selegiline. Accordingly, the buccal film formulation with the poly(lactideco-glycolide) (PLGA) nanospheres of the selegiline was developed. By evaluation of in vitro and in vivo studies, buccal films prepared with selegiline-loaded nanospheres have been observed to show great properties such as good physical properties, sufficient bioadhesion, and controlled drug release. Besides, thanks to the formulation prepared, it was seen that a higher amount of selegiline could be administered through the buccal mucosa. With this study, it is supported that buccal administration of the selegiline is an advantageous and promising approach that can overcome the problems limiting the successful delivery of this drug. ${ }^{56}$

Mishra et al. ${ }^{24}$ developed a nanolipid carrier (NLC) formulation with selegiline hydrochloride to be administered nasally, considering that the nasal route is a convenient way to target the drug directly to the brain. NLC formulation loaded with selegiline hydrochloride showed $93 \pm 5.25 \%$ entrapment efficiency and $51.96 \%$ loading capacity. It has been shown that with the optimized NLC formulation, $70 \%$ release can be achieved within $10 \mathrm{~h}$, and then the drug release continues for up to $22 \mathrm{~h}$ (97\%). The drug was found to improve behavioral parameters in rotenone-induced rats.

Ravi et al. ${ }^{57}$ have developed a nasal thermosensitive gel formulation to provide effective treatment of PD by considering the low oral bioavailability of rasagiline mesylate. Because of pharmacokinetic studies in rabbits, in situ gels were found to provide a significant increase in the bioavailability of rasagiline mesylate.

Çelik et al. ${ }^{58}$ have developed buccal mucoadhesive tablets to increase the low bioavailability of piribedil and provide a controlled release treatment for PD. Generally, buccal tablets prepared with hydroxypropyl methylcellulose can provide the necessary controlled release and physical properties. Because of the study, it was concluded that buccal mucoadhesive tablets provide various advantages such as controlled-release compared to traditional oral dosage forms. It is thought that side effects can be reduced because of the high bioavailability with lower doses to provide the desired effect.

The use of drugs targeted to the brain continuously and safely in PD is critical in the treating of this disease. In a study, surface-modified biodegradable PEG-PLGA nanoparticles were prepared with lactoferrin ( $L f$ ) to target rotigotine intranasally to the brain for PD treatment. When all the results of the study were examined, Lf nanoparticles were shown to be a suitable carrier for targeting rotigotine to the brain intranasally in PD treatment. ${ }^{59}$ 


\section{Developed non-oral PD formulations}

Levodopa/carbidopa intestinal gel (Duopa ${ }^{T M}$ ) (LCIG)

Although L-DOPA is the gold standard in PD treatment, because of its short plasma half-life, oral L-DOPA treatment cannot effectively stimulate receptors. Motor fluctuations are seen due to insufficient plasma level. ${ }^{60} \mathrm{LCIG}$ formulation has been developed to be used to provide a continuous effect by keeping the plasma level of L-DOPA constant. ${ }^{61}$ With the help of this pump, small doses of L-DOPA/carbidopa are administered into the small intestine at regular intervals, by passing the stomach. LCIG allows safe titration of L-DOPA to high doses, even more than $2000 \mathrm{mg} /$ day and leads to more stable L-DOPA plasma concentrations. ${ }^{61}$ Through this formulation, irregular absorption of L-DOPA caused by prolonged GE time in patients with PD is prevented. ${ }^{61}$ In a study, when evaluating the effectiveness of the LCIG formulation against L-DOPA-carbidopa tablets, it was reported that LCIG significantly reduced "OFF" times and increased "ON" time without troubling dyskinesias. Because of the study, percentages of the patients, who were reported as "better" for dyskinesia, tremor, and gait disturbance called motor symptoms, were $80 \%, 55 \%, 65 \%$, and $85 \%$, respectively. Percentages reported for non-motor symptoms, pain, sleep disorders, depression, and incontinence were $50 \%, 50 \%$, $42.5 \%$, and $32.5 \%$, respectively. Studies have shown that LCIG formulation is a promising alternative for advanced patients with PD with motor complications. ${ }^{62}$

\section{Intrajejunal TriGel infusion (LECIG)}

TriGel is a novel formulation obtained by adding entacapone to LCIG. Entacapone reduces conversion of L-DOPA to 3-O-methyldopa by blocking the second-largest pathway of L-DOPA. Thus, the plasma concentration of L-DOPA increases. ${ }^{63}$ In a clinical study, LCIG and LECIG treatments were compared. Because of this study, dose-adjusted L-DOPA exposure was found to be significantly higher in the LECIG formulation compared to LCIG. It was observed that 3 patients had a $20 \%$ increase in systemic exposure to L-DOPA and a $40 \%$ or higher increase in six patients, and 2 patients could not achieve the target systemic exposure. ${ }^{64}$ It is thought that the combination of opicapone, a newly developed COMT inhibitor, and LCIG can provide a similar effect. ${ }^{65}$

\section{Inhaled levodopa powder (Inbrija ${ }^{\circledR}$ )}

L-DOPA inhalation powder (Inbrija ${ }^{\circledR}$ ) is a dry powder formulation administered orally with an inhaler, enabling rapid drug absorption in the pulmonary system. It is manufactured by Acorda Therapeutics and has been approved by FDA to treat the symptoms of Parkinson's patients during "OFF" periods. ${ }^{66}$ Each capsule contains $42 \mathrm{mg}$ spray-dried L-DOPA powder, 1,2-dipalmitoyl-snglycero-3-phosphocholine, and sodium chloride. The dry powder particles (5-10 $\mu \mathrm{m}$ diameter) are homogeneous, low in density, and highly porous for aerosolizability and lung deposition. Inbrija ${ }^{\circledR}$ was developed to achieve a rapid effect by providing a consistent and rapid increase in the concentration of the drug in the bloodstream. Pulmonary administration provides rapid absorption of L-DOPA due to its large surface area and low metabolic activity, so delayed "in" period or dose failures can be avoided. ${ }^{66}$ Because of a study to determine the pharmacokinetics and tolerability of the formulation, $T_{\max }$ was $15 \mathrm{~min}$ in patients who are administered inhaler L-DOPA powder, but after oral administration, $T_{\max }$ ranged from $20 \mathrm{~min}$ to $90 \mathrm{~min}$. However, no changes in lung function parameters were observed in patients and no patient complained of cough or shortness of breath. $.7,68$

\section{Rotigotine patch}

The transdermal patch formulation of rotigotine (Neupro ${ }^{\circledast}$, a dopamine agonist, has been developed for use alone in the early stages of PD or in addition to L-DOPA in the advanced stage of the disease. Rotigotin transdermal patch has been approved in EU, China, and Japan as a combination therapy with monotherapy and L-DOPA for early PD treatment. With the developed transdermal patch formulation, stable rotigotin plasma levels could be achieved for 1-2 days with a single daily administration. ${ }^{69} \mathrm{~A}$ double-blind, placebo-controlled, and randomized study demonstrated that rotigotin patch can well manage both motor function and sleep problems in patients with $\mathrm{PD}$ with motor dysfunction, when waking up in the morning. ${ }^{70}$ Additionally, other important effects of rotigotine patch on nonmotor symptoms include pain, mood, and anhedonia associated with dopamine fluctuations. ${ }^{71}$ Compared with rotigotine patch and other conventional oral dopamine agonists, impulse control disorder was reported to be less common with the use of rotigotine patch. It has been reported that the most common side effects after the application of the rotigotin patch are skin reactions in the application area and some neuropsychiatric problems. ${ }^{71,72}$

\section{Subcutaneous rotigotine-polyoxazoline}

We provided continuous dopaminergic stimulation by preparing a subcutaneous formulation of rotigotine with polyoxazolines. ${ }^{73}$ In vivo studies using rat models with 6-hydroxydopamine lesions have shown that rotigotine-polyoxazoline slow-release conjugate relieves motor symptoms by repeated dosing and provides a long rotigotin half-life. With these promising results, a slow-release conjugate of rotigotine has FDA confirmation to enter phase 1 study (NCT02579473) with anew patients with PD. ${ }^{73}$ Olanow et al. ${ }^{74}$ evaluated the safety, tolerability, and pharmacokinetics of polymer-conjugated rotigotine in patients with PD with a multicentre open-label, multiple incremental, and dose-spaced cohort studies. Because of this study, it has been observed that, when the polymer-conjugated rotigotine is subcutaneously administered once a week, relatively constant plasma rotigotine levels can be achieved, which are safe and well-tolerated.

\section{Subcutaneous apomorphine}

Subcutaneous apomorphine has been developed to manage unpredictable and predictable "OFF" periods, in patients with PD well. The subcutaneous apomorphine has been developed in two different formulations. These are intermittent injection of apomorphine and a continuous infusion of apomorphine with a removable infusion pump without surgery. It is specified that it 
is a very suitable formulation to prevent delayed or failed "ON" situations caused by GE and L-DOPA absorption problems and to alleviate early dystonia or akinesia quickly and safely. It has been reported that a consistent antiparkinsonian response with subcutaneous apomorphine was obtained and no significant circadian changes were observed during this response. The effect of subcutaneous apomorphine injection on "ON" time was evaluated by a multicentre and open-label phase IV study in patients with PD with morning akinesia. In this study, firstly, the normal morning dose of oral L-DOPA was applied to the patients and "ON" times were recorded. Then, "ON" times of the patients were recorded again for a week using apomorphine injection instead of oral L-DOPA. Because of the study, it was observed that apomorphine injection shortened $37 \mathrm{~min}$ the patients' become "ON" status by compared to oral L-DOPA. With several open-label clinical trials, apomorphine infusion significantly reduced OFF time by up to $85 \%$ compared with baseline and increase $\mathrm{ON}$ time by an average of $5.5 \mathrm{~h}$ daily in patients with PD. ${ }^{75,76}$

\section{Inhaled apomorphine (VRO40)}

In PD, it has been observed that "OFF" periods can be managed with subcutaneous apomorphine, but some patients may experience difficulty in application because it requires the injection. For this reason, it is thought that inhaled apomorphine may be useful. To determine optimal efficacy, safety, and tolerability for inhaled apomorphine in patients with PD, randomized, double-blind, active, and placebo parallel-group, and increased dose titration studies were conducted in 16 centers in 3 countries. Because of this study, the meantime to "ON" in 33 patients in the OFF period was found to be $8.1 \mathrm{~min}$ for inhaled apomorphine and $13.1 \mathrm{~min}$ for placebo. Additionally, the proportion of those who became "open" within $40 \mathrm{~min}$ in patients who received inhaled apomorphine and placebo (except "partially open") was found to be $60.0 \%$ and $26.7 \%$, respectively. In the double-blind phase 2 study, tolerability, safety, and effectiveness of VRO40 were evaluated. It was reported that development of a UPDRS III in 47 patients was 26.8 points for inhaled apomorphine and 14.9 for placebo. ${ }^{77,78}$

\section{Sublingual apomorphine (APL-130277)}

APL-130277 is a film strip in clinical development that is investigated for treating OFF periods. It consists of a thin bilayered film designed to improve apomorphine delivery, while optimizing tissue compatibility and film disintegration. The first layer consists of apomorphine and is designed to provide stability, rapid drug diffusion and enhanced bioavailability. The second layer is a buffer layer that is designed to increase drug permeability and neutralize acid formation following drug absorption. As a result, it is designed as a "turning ON" medication to acutely manage OFF episodes by rapidly delivering apomorphine from the oral cavity without any mucosal irritation. Hauser et al. ${ }^{79}$ conducted a phase 2, openlabel, proof-of-concept study to assess tolerability, safety, and efficacy, and to determine the effective doses.

\section{Buccal selegiline (Zydis ${ }^{T M}$ ZELAPAR)}

Non-oral alternative formulations have been explored because of the low oral bioavailability of selegiline, high rate of firstpass effects, and conversion to undesired metabolites in the liver. One of these factors is the tablet formulation prepared for application to buccal mucosa the Zydis ${ }^{\mathrm{TM}}$ technology. ${ }^{80}$ Because of pharmacokinetic studies, Zydis ${ }^{\mathrm{TM}}$ selegiline can inhibit MAO-B at one-eighth of the traditional oral dose and reduce amphetamine metabolites by $80-90 \%$. Because of phase 4 studies, it was seen that ZELAPAR was preferred by patients because it was well tolerated and provides ease of use. ${ }^{25,81}$ Waters et al. ${ }^{81}$ evaluated the safety and efficacy of zydis selegiline in patients with $\mathrm{PD}$ with motor fluctuations during L-DOPA therapy with a short-term clinical study. Because of the study, it was seen that an orodispersible tablet of selegiline as an additional treatment to L-DOPA in patients with PD with motor fluctuation problems was effective and safe.

\section{CONCLUSION}

Current therapy options for PD remain focused on the symptomatic improvement of motor features related predominantly to the loss of dopaminergic neurons in substantia nigra, but do not address the root cause of the disease. Improvements in trial design must evaluate candidate drugs more appropriately, perhaps with the introduction of validated clinical markers. Physicians need practical guidance both to help patients make a judgment on what drug to use and when to initiate it. This remains very much an individual decision and will need to take account of many factors, including the patient's age and co-morbidity and the physician's own interpretation of the data available and the information presented here. Oral dopaminergic treatments were mainly focused on the management of PD symptoms. However, it has been thought and investigated that Gl problems in patients with PD can significantly affect the effectiveness of oral treatments. As a result, it has been observed that Gl problems such as dysphagia, delayed GE, SIBO, and changes in colon motility complicate oral treatment in PD and cause delayed "in" or early morning "OFF" fluctuations in patients. For this reason, nonoral drug delivery systems have been studied to manage PD symptoms effectively.

We seek to bring further clarity to the non-oral treatment options for patients at different stages of PD. The therapies included in this review have all been shown to result in significant improvements of both motor and non-motor symptoms, but each therapy also has many characteristic advantages and drawbacks that need to be matched with the patient's symptomatology.

The costs related to all non-oral drug delivery systems are significant, and further cost reductions are required to increase access to these therapies. Moreover, there is a need for further development of the non-oral continuous drug delivery techniques-both to increase their ease of use and to reduce the relatively frequent device-related adverse effects. In addition to changing the existing drug administration systems, 
new methods of administration are required by examining the current studies.

\section{ACKNOWLEDGEMENTS}

This study was supported by the Scientific and Technological Research Council of Türkiye (grant TÜBITAK- 219S545).

\section{Ethics}

Peer-review: Externally peer-reviewed.

\section{Authorship Contributions}

Concept: M.G., S.Y.K., Design: M.G., S.Y.K., Literature Search: M.G., S.Y.K., Writing: M.G., S.Y.K.

Conflict of Interest: No conflict of interest was declared by the authors.

Financial Disclosure: The authors declared that this study received no financial support.

\section{REFERENCES}

1. Balestrino R, Schapira AHV. Parkinson disease. Eur J Neurol. 2020;27:27-42

2. What is Parkinson's? I American Parkinson Disease Assoc. Published 2020. Accessed: March 18, 2020. Available from: https://www. apdaparkinson.org/what-is-parkinsons/

3. Pfeiffer RF. Non-motor symptoms in Parkinson's disease. Parkinsonism Relat Disord. 2016;22(Suppl 1):S119-S122.

4. Stocchi F. The hypothesis of the genesis of motor complications and continuous dopaminergic stimulation in the treatment of Parkinson's disease. Parkinsonism Relat Disord. 2009;15(Suppl 1):S9-S15.

5. Connolly BS, Lang AE. Pharmacological treatment of Parkinson disease: a review. JAMA. 2014;311:1670-1683.

6. Price J, Martin H, Ebenezer L. The use of non-oral therapies in Parkinson's disease. J Prescr Prac. 2018;16:26-30.

7. Chapuis S, Ouchchane L, Metz O, Gerbaud L, Durif F. Impact of the motor complications of Parkinson's disease on the quality of life. Mov Disord. 2005;20:224-230.

8. Rizos A, Martinez-Martin P, Odin P, Antonini A, Kessel B, Kozul TK, Todorova A, Douiri A, Martin A, Stocchi F, Dietrichs E, Chaudhuri KR; EUROPAR and the IPDMDS non-Motor PD Study Group. Characterizing motor and non-motor aspects of early-morning off periods in Parkinson's disease: an international multicenter study. Parkinsonism Relat Disord. 2014:20:1231-1235.

9. Fasano A, Visanji NP, Liu LW, Lang AE, Pfeiffer RF. Gastrointestinal dysfunction in Parkinson's disease. Lancet Neurol. 2015;14:625-639.

10. What is Parkinson's? | European Parkinson's Disease AssociationAccessed March 28, 2021. Available from: https://www.epda. eu.com/about-parkinsons/what-is-parkinsons/

11. Salat D, Tolosa E. Levodopa in the treatment of Parkinson's disease: current status and new developments. J Parkinsons Dis. 2013;3:255-269.

12. Freitas ME, Hess CW, Fox SH. Motor complications of dopaminergic medications in Parkinson's disease. Semin Neurol. 2017;37:147-157.

13. van Laar T, Borgemeester R. The need for non-oral therapy in Parkinson's disease; a potential role for apomorphine. Parkinsonism Relat Disord. 2016;33(Suppl 1):S22-S27.
14. Ozlece HK, Guvendi GF, Huseyinoglu N, Ehi Y, Kivrak Y. Cytological and cytometric analysis of oral mucosa in patients with Alzheimer's and Parkinson's disease. Neuropsychiatr Dis Treat. 2018;14:1901-1906.

15. Stocchi $F$, Jenner $P$, Obeso JA. When do levodopa motor fluctuations first appear in Parkinson's disease? Eur Neurol. 2010;63:257-266.

16. Marrinan S, Emmanuel AV, Burn DJ. Delayed gastric emptying in Parkinson's disease. Mov Disord. 2014;29:23-32.

17. Pfeiffer RF. Gastrointestinal dysfunction in Parkinson's disease. Lancet Neurol. 2003;2:107-116.

18. Goldenberg MM. Medical management of Parkinson's disease. P T. 2008;33:590-606.

19. Ahlskog JE. Slowing Parkinson's disease progression: recent dopamine agonist trials. Neurology. 2003;60:381-389.

20. Kaakkola S. Problems with the present inhibitors and a relevance of new and improved COMT inhibitors in Parkinson's disease. Int Rev Neurobiol. 2010;95:207-225

21. Kaakkola S. Clinical pharmacology, therapeutic use and potential of COMT inhibitors in Parkinson's disease. Drugs. 2000;59:1233-1250.

22. Espinoza S, Manago F, Leo D, Sotnikova TD, Gainetdinov RR. Role of catechol-O-methyltransferase (COMT)-dependent processes in Parkinson's disease and L-DOPA treatment. CNS Neurol Disord Drug Targets. 2012;11:251-263.

23. DeMaagd G, Philip A. Parkinson' disease and its management: part 3: nondopaminergic and nonpharmacological treatment options. P T. 2015; $40: 668-679$

24. Mishra N, Sharma S, Deshmukh R, Kumar A, Sharma R. Development and characterization of nasal delivery of selegiline hydrochloride loaded nanolipid carriers for the management of Parkinson's disease. Cent Nerv Syst Agents Med Chem. 2019;19:46-56.

25. Clarke A, Johnson ES, Mallard N, Corn TH, Johnston A, Boyce M, Warrington S, MacMahon DG. A new low-dose formulation of selegiline: clinical efficacy, patient preference and selectivity for MAO-B inhibition. J Neural Transm (Vienna). 2003;110:1257-1271.

26. Lee KC, Chen JJ. Transdermal selegiline for the treatment of major depressive disorder. Neuropsychiatr Dis Treat. 2007;3:527-537.

27. Müller T, Hoffmann JA, Dimpfel W, Oehlwein C. Switch from selegiline to rasagiline is beneficial in patients with Parkinson's disease. J Neural Transm (Vienna). 2013;120:761-765.

28. Finberg JP, Gillman K. Selective inhibitors of monoamine oxidase type B and the "cheese effect". Int Rev Neurobiol. 2011;100:169-190.

29. Goren T, Adar L, Sasson N, Weiss YM. Clinical pharmacology tyramine challenge study to determine the selectivity of the monoamine oxidase type B (MAO-B) inhibitor rasagiline. J Clin Pharmacol. 2010;50:1420-1428.

30. deMarcaida JA, Schwid SR, White WB, Blindauer K, Fahn S, Kieburtz K, Stern M, Shoulson I; Parkinson Study Group TEMPO; PRESTO Tyramine Substudy Investigators and Coordinators. Effects of tyramine administration in Parkinson's disease patients treated with selective MAO-B inhibitor rasagiline. Mov Disord. 2006;21:1716-1721.

31. Müller T. Experimental dopamine reuptake inhibitors in Parkinson's disease: a review of the evidence. J Exp Pharmacol. 2021;13:397-408.

32. Pagliaro LA, Pagliaro AM. Levodopa [L-Dopa]. In: PNDR: Psychologists' neuropsychotropic drug reference. Routledge; 2020:185-189.

33. Suttrup I, Warnecke T. Dysphagia in Parkinson's disease. Dysphagia. 2016;31:24-32. 
34. Kalf JG, de Swart BJ, Bloem BR, Munneke M. Prevalence of oropharyngeal dysphagia in Parkinson's disease: a meta-analysis. Parkinsonism Relat Disord. 2012;18:311-315.

35. Aydogdu I, Tanriverdi Z, Ertekin C. Dysfunction of bulbar central pattern generator in ALS patients with dysphagia during sequential deglutition. Clin Neurophysiol. 2011;122:1219-1228.

36. Lam K, Lam FK, Lau KK, Chan YK, Kan EY, Woo J, Wong FK, Ko A. Simple clinical tests may predict severe oropharyngeal dysphagia in Parkinson's disease. Mov Disord. 2007;22:640-644.

37. Leibner J, Ramjit A, Sedig L, Dai Y, Wu SS, Jacobson C, Okun MS, Rodriguez RL, Malaty IA, Fernandez HH. The impact of and the factors associated with drooling in Parkinson's disease. Parkinsonism Relat Disord. 2010;16:475-477.

38. Müller B, Larsen JP, Wentzel-Larsen T, Skeie GO, Tysnes OB; Parkwest Study Group. Autonomic and sensory symptoms and signs in incident, untreated Parkinson's disease: frequent but mild. Mov Disord. 2011;26:6572.

39. Doi H, Sakakibara R, Sato M, Masaka T, Kishi M, Tateno A, Tateno F, Tsuyusaki Y, Takahashi O. Plasma levodopa peak delay and impaired gastric emptying in Parkinson's disease. J Neurol Sci. 2012;319:86-88.

40. Tanaka Y, Kato T, Nishida H, Yamada M, Koumura A, Sakurai T, Hayashi Y, Kimura A, Hozumi I, Araki H, Murase M, Nagaki M, Moriwaki H, Inuzuka $\mathrm{T}$. Is there a delayed gastric emptying of patients with early-stage, untreated Parkinson's disease? An analysis using the 13C-acetate breath test. J Neurol. 2011;258:421-426.

41. Goetze O, Wieczorek J, Mueller T, Przuntek H, Schmidt WE, Woitalla D. Impaired gastric emptying of a solid test meal in patients with Parkinson's disease using 13C-sodium octanoate breath test. Neurosci Lett. 2005;375:170-173.

42. Unger MM, Möller JC, Mankel K, Schmittinger K, Eggert KM, Stamelou M, Stiasny-Kolster K, Bohne K, Bodden M, Mayer G, Oertel WH, Tebbe JJ. Patients with idiopathic rapid-eye-movement sleep behavior disorder show normal gastric motility assessed by the 13C-octanoate breath test. Mov Disord. 2011;26:2559-2563.

43. Tan AH, Mahadeva S, Thalha AM, Gibson PR, Kiew CK, Yeat CM, Ng SW, Ang SP, Chow SK, Tan CT, Yong HS, Marras C, Fox SH, Lim SY. Small intestinal bacterial overgrowth in Parkinson's disease. Parkinsonism Relat Disord. 2014;20:535-540.

44. Fasano A, Bove F, Gabrielli M, Petracca M, Zocco MA, Ragazzoni E, Barbaro F, Piano C, Fortuna S, Tortora A, Di Giacopo R, Campanale M, Gigante G, Lauritano EC, Navarra P, Marconi S, Gasbarrini A, Bentivoglio AR. The role of small intestinal bacterial overgrowth in Parkinson's disease. Mov Disord. 2013;28:1241-1249.

45. Cheon SM, Ha MS, Park MJ, Kim JW. Nonmotor symptoms of Parkinson's disease: prevalence and awareness of patients and families. Parkinsonism Relat Disord. 2008;14:286-290.

46. Gabrielli M, Bonazzi P, Scarpellini E, Bendia E, Lauritano EC, Fasano A, Ceravolo MG, Capecci M, Rita Bentivoglio A, Provinciali L, Tonali PA, Gasbarrini A. Prevalence of small intestinal bacterial overgrowth in Parkinson's disease. Mov Disord. 2011;26:889-892.

47. Ray Chaudhuri K, Qamar MA, Rajah T, Loehrer P, Sauerbier A, Odin P, Jenner P. Non-oral dopaminergic therapies for Parkinson's disease: current treatments and the future. NPJ Parkinsons Dis. 2016;2:16023.

48. Lomax AE, Linden DR, Mawe GM, Sharkey KA. Effects of gastrointestinal inflammation on enteroendocrine cells and enteric neural reflex circuits. Auton Neurosci. 2006;126-127:250-257.
49. Chaná $P$, Kuntsmann $C$, Reyes-Parada M, Sáez-Briones P. Delayed early morning turn "ON" in response to a single dose of levodopa in advanced Parkinson's disease: pharmacokinetics should be considered. J Neurol Neurosurg Psychiatry. 2004;75:1782-1783.

50. Forsyth CB, Shannon KM, Kordower JH, Voigt RM, Shaikh M, Jaglin JA, Estes JD, Dodiya HB, Keshavarzian A. Increased intestinal permeability correlates with sigmoid mucosa alpha-synuclein staining and endotoxin exposure markers in early Parkinson's disease. PLoS One. 2011;6:e28032.

51. van Kessel SP, Frye AK, El-Gendy AO, Castejon M, Keshavarzian A, van Dijk G, El Aidy S. Gut bacterial tyrosine decarboxylases restrict levels of levodopa in the treatment of Parkinson's disease. Nat Commun. 2019;10:310.

52. Hill-Burns EM, Debelius JW, Morton JT, Wissemann WT, Lewis MR, Wallen ZD, Peddada SD, Factor SA, Molho E, Zabetian CP, Knight R, Payami H. Parkinson's disease and Parkinson's disease medications have distinct signatures of the gut microbiome. Mov Disord. 2017;32:739749.

53. Priano L, Albani G, Brioschi A, Calderoni S, Lopiano L, Rizzone M, Cavalli R, Gasco MR, Scaglione F, Fraschini F, Bergamasco B, Mauro A. Transdermal apomorphine permeation from microemulsions: a new treatment in Parkinson's disease. Mov Disord. 2004;19:937-942.

54. Rascol O, Azulay JP, Blin O, Bonnet AM, Brefel-Courbon C, Césaro P, Damier P, Debilly B, Durif F, Galitzky M, Grouin JM, Pennaforte S, Villafane G, Yaici S, Agid Y. Orodispersible sublingual piribedil to abort OFF episodes: a single dose placebo-controlled, randomized, doubleblind, cross-over study. Mov Disord. 2010;25:368-376.

55. Sintov AC, Levy HV, Greenberg I. Continuous transdermal delivery of L-DOPA based on a self-assembling nanomicellar system. Pharm Res. 2017;34:1459-1468.

56. Al-Dhubiab BE, Nair AB, Kumria R, Attimarad M, Harsha S. Development and evaluation of buccal films impregnated with selegiline-loaded nanospheres. Drug Deliv. 2016;23:2154-2162.

57. Ravi PR, Aditya N, Patil S, Cherian L. Nasal in-situ gels for delivery of rasagiline mesylate: improvement in bioavailability and brain localization. Drug Deliv. 2015;22:903-910.

58. Çelik B, Özdemir S, Barla Demirkoz A, Üner M. Optimization of piribedil mucoadhesive tablets for efficient therapy of Parkinson's disease: physical characterization and ex vivo drug permeation through buccal mucosa. Drug Dev Ind Pharm. 2017;43:1836-1845.

59. Bi C, Wang A, Chu Y, Liu S, Mu H, Liu W, Wu Z, Sun K, Li Y. Intranasal delivery of rotigotine to the brain with lactoferrin-modified PEG-PLGA nanoparticles for Parkinson's disease treatment. Int J Nanomedicine. 2016;11:6547-6559.

60. Stocchi F, Tagliati M, Olanow CW. Treatment of levodopa-induced motor complications. Mov Disord. 2008;23(Suppl 3):S599-S612.

61. Wang L, Li J, Chen J. Levodopa-carbidopa intestinal gel in Parkinson's disease: a systematic review and meta-analysis. Front Neurol. 2018;9.

62. Gültekin M, Ulukan C,, Tezcan S, Doğu O, Hanağasi H, Bilgiç B, Bora Tokçaer A, Çakmur R, Elibol B, Mirza M, İnce Günal D, Erer Özbek ÇS, Kenangil G, Yilmaz Küsbeci Ö, Akbostanci MC. Multicenter study of levodopa carbidopa intestinal gel in Parkinson's disease: the Turkish experience. Turk J Med Sci. 2020;50:66-85.

63. Senek M, Nielsen El, Nyholm D. Levodopa-entacapone-carbidopa intestinal gel in Parkinson's disease: a randomized crossover study. Mov Disord. 2017;32:283-286. 
64. Nyholm D, Johansson A, Lennernäs $H$, Askmark H. Levodopa infusion combined with entacapone or tolcapone in Parkinson disease: a pilot trial. Eur J Neurol. 2012;19:820-826.

65. Ferreira JJ, Lees A, Rocha JF, Poewe W, Rascol O, Soares-da-Silva $\mathrm{P}$; Bi-Park 1 investigators. Opicapone as an adjunct to levodopa in patients with Parkinson's disease and end-of-dose motor fluctuations: a randomised, double-blind, controlled trial. Lancet Neurol. 2016;15:154165.

66. Drug Approval Package: Inbrija.

67. Lipp MM, Batycky R, Moore J, Leinonen M, Freed MI. Preclinical and clinical assessment of inhaled levodopa for OFF episodes in Parkinson's disease. Sci Transl Med. 2016;8:360ra136.

68. Luinstra M, Rutgers W, van Laar T, Grasmeijer F, Begeman A, Isufi V, Steenhuis L, Hagedoorn P, de Boer A, Frijlink HW. Pharmacokinetics and tolerability of inhaled levodopa from a new dry-powder inhaler in patients with Parkinson's disease. Ther Adv Chronic Dis. 2019;10:2040622319857617.

69. Frampton JE. Rotigotine transdermal patch: a review in Parkinson's disease. CNS Drugs. 2019;33:707-718.

70. Trenkwalder C, Kies B, Rudzinska M, Fine J, Nikl J, Honczarenko K, Dioszeghy P, Hill D, Anderson T, Myllyla V, Kassubek J, Steiger M, Zucconi M, Tolosa E, Poewe W, Surmann E, Whitesides J, Boroojerdi B, Chaudhuri KR; Recover Study Group. Rotigotine effects on early morning motor function and sleep in Parkinson's disease: a doubleblind, randomized, placebo-controlled study (RECOVER). Mov Disord. 2011;26:90-99.

71. Rizos A, Sauerbier A, Antonini A, Weintraub D, Martinez-Martin P, Kessel B, Henriksen T, Falup-Pecurariu C, Silverdale M, Durner G, Røkenes Karlsen K, Grilo M, Odin P, Chaudhuri KR; EUROPAR and the IPMDS Non-Motor-PD-Study Group. A European multicentre survey of impulse control behaviours in Parkinson's disease patients treated with shortand long-acting dopamine agonists. Eur J Neurol. 2016;23:1255-1261.

72. Ray Chaudhuri K, Martinez-Martin P, Antonini A, Brown RG, Friedman $\mathrm{JH}$, Onofrj M, Surmann E, Ghys L, Trenkwalder C. Rotigotine and specific non-motor symptoms of Parkinson's disease: post hoc analysis of RECOVER. Parkinsonism Relat Disord. 2013;19:660-665.
73. Eskow Jaunarajs KL, Standaert DG, Viegas TX, Bentley MD, Fang Z, Dizman B, Yoon K, Weimer R, Ravenscroft P, Johnston TH, Hill MP, Brotchie JM, Moreadith RW. Rotigotine polyoxazoline conjugate SER214 provides robust and sustained antiparkinsonian benefit. Mov Disord. 2013;28:1675-1682.

74. Olanow CW, Standaert DG, Kieburtz K, Viegas TX, Moreadith R. Onceweekly subcutaneous delivery of polymer-linked rotigotine (SER-214) provides continuous plasma levels in Parkinson's disease patients. Mov Disord. 2020;35:1055-1061.

75. Isaacson S, Lew M, Ondo W, Hubble J, Clinch T, Pagan F. Apomorphine subcutaneous injection for the management of morning akinesia in Parkinson's disease. Mov Disord Clin Pract. 2017;4:78-83.

76. Deleu D, Hanssens Y, Northway MG. Subcutaneous apomorphine: an evidence-based review of its use in Parkinson's disease. Drugs Aging. 2004;21:687-709.

77. Grosset KA, Malek N, Morgan F, Grosset DG. Inhaled apomorphine in patients with 'on-off' fluctuations: a randomized, double-blind, placebocontrolled, clinic and home based, parallel-group study. J Parkinsons Dis. 2013;3:31-37.

78. Grosset KA, Malek N, Morgan F, Grosset DG. Inhaled dry powder apomorphine (VR040) for 'off' periods in Parkinson's disease: an inclinic double-blind dose ranging study. Acta Neurol Scand. 2013;128:166171.

79. Hauser RA, Olanow CW, Dzyngel B, Bilbault T, Shill H, Isaacson S, Dubow J, Agro A. Sublingual apomorphine (APL-130277) for the acute conversion of OFF to ON in Parkinson's disease. Mov Disord. 2016;31:1366-1372.

80. Clarke A, Brewer F, Johnson ES, Mallard N, Hartig F, Taylor S, Corn TH. A new formulation of selegiline: improved bioavailability and selectivity for MAO-B inhibition. J Neural Transm (Vienna). 2003;110:1241-1255.

81. Waters CH, Sethi KD, Hauser RA, Molho E, Bertoni JM; Zydis Selegiline Study Group. Zydis selegiline reduces off time in Parkinson's disease patients with motor fluctuations: a 3-month, randomized, placebocontrolled study. Mov Disord. 2004;19:426-432. 